\title{
Prime sequences
}

\author{
J. V. Leyendekkers ${ }^{1}$ and A. G. Shannon ${ }^{2}$ \\ ${ }^{1}$ Faculty of Science, The University of Sydney, NSW 2006, Australia \\ e-mail: jeanvaldek@gmail.com \\ ${ }^{2}$ Warrane College, The University of New South Wales, NSW 2033, Australia \\ Emeritus Professor, University of Technology Sydney, NSW 2007, Australia \\ e-mail: tshannon38@gmail.com
}

Received: 8 May 2018

Accepted: 30 August 2018

\begin{abstract}
Primes are considered in three sequences, of which two are exclusive to specific primes. These sequences have the integers represented in the form $n R$ where $R$ is the right-enddigit of the prime and $n$ represents the remaining left digits which are given by linear equations. Keywords: Right-end-digits, Integer structure analysis, Modular rings, Prime-indexed numbers, Fibonacci numbers, Mersenne numbers.
\end{abstract}

2010 Mathematics Subject Classification: 11A51, 11 A07.

\section{Introduction}

An unexpected bias in the distribution of consecutive primes [2] is clearly apparent in the rightend-digit (RED) considerations of their distributions [3-10]. Three main sequences of primes occur when presented in the form $n R$, where $R$ is the RED of the prime and $n$ represents the remaining left digits; for example, for the prime 177, $n=17$ and $R=7$. Thus RED-defined sequences are embedded in three principal formats:

$$
\begin{gathered}
n=3 t+2 \\
n=3 t \\
n=3 t+1
\end{gathered}
$$

The aim of this paper is to consider the non-randomness of these sequences of RED-defined primes; this is also illustrated with modular rings and integer sequence analysis [3-10]. 


\section{The sequence $n=3 t+2$}

For this sequence, $R=1$ or 7 will always yield a composite integer value, but a prime integer value is possible if their RED is 3 or 9 (see Table 1), which appear to have a high prime 'yield'.

\begin{tabular}{|c|c|c|c|c|c|c|c|c|c|c|c|c|c|}
\hline $\boldsymbol{n}$ & $\mathbf{2}$ & $\mathbf{5}$ & $\mathbf{8}$ & $\mathbf{1 1}$ & $\mathbf{1 4}$ & $\mathbf{1 7}$ & $\mathbf{2 0}$ & $\mathbf{2 3}$ & $\mathbf{2 6}$ & $\mathbf{2 9}$ & $\mathbf{3 2}$ & $\mathbf{3 5}$ & $\mathbf{3 8}$ \\
\hline $\boldsymbol{t}$ & $\mathbf{0}$ & $\mathbf{1}$ & $\mathbf{2}$ & $\mathbf{3}$ & $\mathbf{4}$ & $\mathbf{5}$ & $\mathbf{6}$ & $\mathbf{7}$ & $\mathbf{8}$ & $\mathbf{9}$ & $\mathbf{1 0}$ & $\mathbf{1 1}$ & $\mathbf{1 2}$ \\
\hline \hline $\boldsymbol{R}=\mathbf{1}$ & 21 & 51 & 81 & 111 & 141 & 171 & 201 & 231 & 261 & 291 & 321 & 351 & 381 \\
\hline type & $c$ & $c$ & $c$ & $c$ & $c$ & $c$ & $c$ & $c$ & $c$ & $c$ & $c$ & $c$ & $c$ \\
\hline \hline $\boldsymbol{R}=\mathbf{7}$ & 27 & 57 & 87 & 117 & 147 & 177 & 207 & 237 & 267 & 297 & 327 & 357 & 387 \\
\hline type & $c$ & $c$ & $c$ & $c$ & $c$ & $c$ & $c$ & $c$ & $c$ & $c$ & $c$ & $c$ & $c$ \\
\hline \hline $\boldsymbol{R}=\mathbf{3}$ & 23 & 53 & 84 & 113 & 143 & 173 & 203 & 233 & 263 & 293 & 323 & 353 & 383 \\
\hline type & $p$ & $p$ & $p$ & $p$ & $c$ & $p$ & $c$ & $p$ & $p$ & $p$ & $c$ & $p$ & $p$ \\
\hline $\boldsymbol{R}=\mathbf{9}$ & 29 & 59 & 89 & 119 & 149 & 179 & 209 & 239 & 269 & 299 & 329 & 359 & 389 \\
\hline type & $p$ & $p$ & $p$ & $c$ & $p$ & $p$ & $c$ & $p$ & $p$ & $c$ & $c$ & $p$ & $p$ \\
\hline
\end{tabular}

Table 1. Primality for $n=3 t+2$ (where $p$ denotes prime, $c$ denotes composite)

Many sequences can obviously be obtained from the $t$ values in equation (1.1). For instance,

$$
n=a+21 j
$$

as illustrated in Table 2 for $a=2+3 i$, in which 3 and 9 REDs of primes are often both produced for a given $n$.

\begin{tabular}{|c|c|c|c|c|}
\hline$a$ & REDs of primes & Range & $\%$ of primes & Total \% of primes \\
\hline \multirow{2}{*}{2} & 3 & $\overline{(23 ; 13883)}$ & 53 & \multirow[b]{2}{*}{76} \\
\hline & 9 & $(29 ; 13679)$ & 52 & \\
\hline \multirow{2}{*}{5} & 3 & $(53 ; 10343)$ & 54 & \multirow{2}{*}{82} \\
\hline & 9 & $(59 ; 10559)$ & 52 & \\
\hline \multirow{2}{*}{8} & 3 & $(83 ; 10163)$ & 50 & \multirow{2}{*}{72} \\
\hline & 9 & $(89 ; 10589)$ & 50 & \\
\hline 11 & 3 & $(113 ; 23003)$ & 46 & 46 \\
\hline \multirow{2}{*}{14} & 3 & $(143 ; 10433)$ & 54 & \multirow{2}{*}{80} \\
\hline & 9 & $(149 ; 8969)$ & 48 & \\
\hline \multirow{2}{*}{17} & 3 & $(173 ; 10463)$ & 62 & \multirow{2}{*}{74} \\
\hline & 9 & $(179 ; 10259)$ & 60 & \\
\hline 20 & 9 & $(419 ; 10709)$ & 56 & 56 \\
\hline \multirow[b]{2}{*}{23} & 3 & $(23 ; 13883)$ & 53 & \multirow{2}{*}{76} \\
\hline & 9 & $(29 ; 13679)$ & 52 & \\
\hline \multirow{2}{*}{26} & 3 & $(53 ; 10343)$ & 54 & \multirow{2}{*}{82} \\
\hline & 9 & $(59 ; 10559)$ & 52 & \\
\hline
\end{tabular}

Table 2. Equation (2.1) with $a=2+3 i$

\section{The sequence $n=3 t$}

Only primes with REDs equal to 1 and 7 are produced, since $n R$ can be divided by 3 . Similar forms of imbedded sequences apply as for $(2+3 t)$ (see Table 3). In this case, $a=3 i$ in equation (2.1). 


\begin{tabular}{|c|c|c|c|c|}
\hline$a$ & REDs of primes & Range & $\%$ of primes & Total \% of primes \\
\hline$\overline{0}$ & 1 & $(211 ; 10501)$ & $\overline{48}$ & $\overline{48}$ \\
\hline \multirow{2}{*}{3} & 1 & $(31 ; 10531)$ & 56 & \multirow{2}{*}{78} \\
\hline & 7 & $(37 ; 9907)$ & 50 & \\
\hline \multirow{2}{*}{6} & 1 & $(61 ; 10141)$ & 68 & \multirow{2}{*}{78} \\
\hline & 7 & $(67 ; 10567)$ & 50 & \\
\hline 9 & 7 & $(97 ; 10597)$ & 48 & 48 \\
\hline \multirow{2}{*}{12} & 1 & $(331 ; 9781)$ & 54 & \multirow{2}{*}{78} \\
\hline & 7 & $(127 ; 10627)$ & 58 & \\
\hline \multirow{2}{*}{15} & 1 & $(151 ; 10651)$ & 46 & \multirow{2}{*}{74} \\
\hline & 7 & $(157 ; 10657)$ & 56 & \\
\hline \multirow{2}{*}{18} & 1 & $(181 ; 9631)$ & 52 & \multirow{2}{*}{80} \\
\hline & 7 & $(397 ; 10687)$ & 56 & \\
\hline 21 & 1 & $(211 ; 10501)$ & 48 & 48 \\
\hline \multirow{2}{*}{24} & 1 & $(31 ; 10531)$ & 56 & \multirow{2}{*}{78} \\
\hline & 7 & $(37 ; 9907)$ & 50 & \\
\hline \multirow{2}{*}{27} & 1 & $(61 ; 10141)$ & 68 & \multirow{2}{*}{78} \\
\hline & 7 & $(67 ; 10567)$ & 50 & \\
\hline
\end{tabular}

Table 3. Equation (2.1) with $a=3 i$

\section{The sequence $n=3 t+1$}

This sequence produces primes with REDs 1, 3, 7 and 9. The embedded sequences produce primes for only three of the REDs for a given $a$. Since $R=5$ can never be prime (except for $n=0$ ), the imbedded sequences can produce primes with all four REDs 1, 3, 7, 9 (Table 4).

\begin{tabular}{|c|c|c|c|c|c|}
\hline $\begin{array}{l}\text { RED that is } \\
\text { always composite } \\
\text { for } n=i+3 t\end{array}$ & $\begin{array}{l}\text { Imbedded } \\
\text { sequences }\end{array}$ & $\begin{array}{c}\text { REDs } \\
\text { forming } \\
\text { primes }\end{array}$ & $R$ & $\begin{array}{c}\text { \% of primes } \\
\text { produced }\end{array}$ & $\begin{array}{c}\text { Total \% } \\
\text { of primes }\end{array}$ \\
\hline \multirow{4}{*}{1} & \multirow{4}{*}{$\begin{array}{c}n=16+21 j \\
t=5+7 i\end{array}$} & \multirow{4}{*}{$3,7,9$} & 1 & 0 & \multirow{4}{*}{93} \\
\hline & & & 3 & 59 & \\
\hline & & & 7 & 54 & \\
\hline & & & 9 & 44 & \\
\hline \multirow{4}{*}{3} & \multirow{4}{*}{$\begin{array}{c}n=13+21 j \\
t=4+7 i\end{array}$} & \multirow{4}{*}{$1,7,9$} & 1 & 35 & \multirow{4}{*}{90} \\
\hline & & & 3 & 0 & \\
\hline & & & 7 & 53 & \\
\hline & & & 9 & 58 & \\
\hline \multirow{5}{*}{5} & \multirow{5}{*}{$\begin{array}{c}n=10+21 j \\
t=3+7 i\end{array}$} & \multirow{5}{*}{$1,3,7,9$} & 1 & 73 & \multirow{5}{*}{95} \\
\hline & & & 3 & 70 & \\
\hline & & & 5 & 0 & \\
\hline & & & 7 & 51 & \\
\hline & & & 9 & 55 & \\
\hline
\end{tabular}

(contd.) 


\begin{tabular}{|c|c|c|c|c|c|}
\hline $\begin{array}{l}\text { RED that is } \\
\text { always composite } \\
\text { for } n=i+3 t\end{array}$ & $\begin{array}{l}\text { Imbedded } \\
\text { sequences }\end{array}$ & $\begin{array}{c}\text { REDs } \\
\text { forming } \\
\text { primes }\end{array}$ & $R$ & $\begin{array}{l}\% \text { of primes } \\
\text { produced }\end{array}$ & $\begin{array}{c}\text { Total \% } \\
\text { of primes }\end{array}$ \\
\hline \multirow{4}{*}{7} & \multirow{4}{*}{$\begin{aligned} n & =7+21 j \\
t & =2+7 i\end{aligned}$} & \multirow{4}{*}{$1,3,9$} & 1 & 50 & \multirow{4}{*}{93} \\
\hline & & & 3 & 51 & \\
\hline & & & 7 & 0 & \\
\hline & & & 9 & 52 & \\
\hline \multirow{4}{*}{9} & \multirow{4}{*}{$\begin{array}{c}n=4+21 j \\
t=1+7 i\end{array}$} & \multirow{4}{*}{$1,3,7$} & 1 & 50 & \multirow{4}{*}{93} \\
\hline & & & 3 & 35 & \\
\hline & & & 7 & 52 & \\
\hline & & & 9 & 0 & \\
\hline
\end{tabular}

Table 4. High percentage of primes in imbedded sequences

\section{5 'Large' primes}

Since the sequences have genuine structural features they should be applicable independently of the size of the integers.

Some examples are set out in Tables 5 and 6 for what one might call large primes.

\begin{tabular}{|c|c|c|c|c|}
\hline Prime & $\boldsymbol{R}$ & Major series & Imbedded series & Remarks \\
\hline 104395301 & 1 & $1+3 t$ & $\begin{aligned} n & =10+21 j \\
j & =497120\end{aligned}$ & $\begin{array}{l}\text { Integers with this } n \& R=3,7 \text { or } 9 \\
\text { could be prime }\end{array}$ \\
\hline 179426111 & 1 & $1+3 t$ & $\begin{array}{l}n=1+21 j \\
j=854410\end{array}$ & $\begin{array}{l}\text { Integers with this } n \& R=3,7 \text { or } 9 \\
\text { could be prime }\end{array}$ \\
\hline 179425033 & 3 & $1+3 t$ & $\begin{aligned} n & =19+21 j \\
j & =854404\end{aligned}$ & $\begin{array}{l}\text { Integers with this } n \& R=1,7 \text { or } 9 \\
\text { could be prime }\end{array}$ \\
\hline 179434483 & 3 & $1+3 t$ & $\begin{aligned} n & =19+21 j \\
j & =854404\end{aligned}$ & $\begin{array}{l}\text { Integers with this } n \& R=7 \\
\text { could be prime }\end{array}$ \\
\hline 179425177 & 7 & $3 t$ & $\begin{array}{l}n=2+21 j \\
j=854405\end{array}$ & $\begin{array}{l}\text { Integers with this } n \& R=1 \\
\text { could be prime }\end{array}$ \\
\hline 179434487 & 7 & $1+3 t$ & $\begin{aligned} n & =19+21 j \\
j & =854449\end{aligned}$ & $\begin{array}{l}\text { Integers with this } n \& R=3 \text {, or } 9 \\
\text { could be prime }\end{array}$ \\
\hline 179425319 & 9 & $2+3 t$ & $\begin{array}{l}n=5+21 j \\
j=854406\end{array}$ & $\begin{array}{l}\text { Integers with this } n \& R=3 \\
\text { could be prime }\end{array}$ \\
\hline 179426369 & 9 & $2+3 t$ & $\begin{array}{l}n=5+21 j \\
j=854411\end{array}$ & $\begin{array}{l}\text { Integer with this } n \& R=3 \\
\text { could be prime }\end{array}$ \\
\hline
\end{tabular}

Table 5. Imbedded series for 'large' primes 


\begin{tabular}{|c|c|c|c|l|}
\hline Prime & $\boldsymbol{R}$ & $\begin{array}{c}\text { Major } \\
\text { series }\end{array}$ & \multicolumn{1}{|c|}{ Imbedded series } & \multicolumn{1}{c|}{ Remarks } \\
\hline \hline 817504253838041641 & 1 & $3 t$ & $\begin{array}{c}n=15+21 j \\
j=3892877399228769\end{array}$ & $\begin{array}{l}\text { Integer with this } n \& R=7 \\
\text { could be prime }\end{array}$ \\
\hline 961748941982451653 & 3 & $2+3 t$ & $\begin{array}{c}n=2+21 j \\
j=4579956866587103\end{array}$ & $\begin{array}{l}\text { Integer with this } n \& R=9 \\
\text { could be prime }\end{array}$ \\
\hline 275604547295075147 & 7 & $1+3 t$ & $\begin{array}{c}n=10+21 j \\
j=1312402606167024\end{array}$ & $\begin{array}{l}\text { Integer with this } n \& R=1,3,9 \\
\text { could be prime }\end{array}$ \\
\hline 593441861613651349 & 9 & $1+3 t$ & $\begin{array}{l}n=1+21 j \\
j=2825913626731673\end{array}$ & $\begin{array}{l}\text { Integer with this } n \& R=1,3,7 \\
\text { could be prime }\end{array}$ \\
\hline
\end{tabular}

Table 6. Imbedded series for 'large' primes

\section{Fibonacci and Mersenne primes}

\subsection{Fibonacci primes}

When $R=3$, composites always occur when $n=2+3 t$ for $p>29$ and there is a bias towards primes for $R=7$ (see Tables 7 and 8 ). Nine primes are formed from $25 p$ values (36\%).

Of the $25 p$ values, twelve yield the Fibonacci number, $F_{p}$, [10] with $n=1+3 t$, six have $n=2+3 t$, and six have $n=3 t$ (see Table 7) [12].

When $n=13+21 j$ or $17+21 j$, all $F_{p}$ are composite (see Table 7), and all $F_{p}$ with $R=1$ are composite for the range in this table. For instance, $F_{13}=1,346,269=557 \times 2417$ : prime subscript but composite number, whereas $F_{29}=514,229$ which is prime: prime subscript and prime number. Similarly with $F_{13}=233$. In this case, $R=3$ as in Table 1 , and $a=2$ with a RED of 3 as in Table 2; that is, $a=n-2 j=23-21$. The search for new near-patterns among primes and prime-indexed numbers goes on with a variety of interesting techniques [1].

\begin{tabular}{|c|r|r|r|r|c|}
\hline $\boldsymbol{p}$ & $\boldsymbol{F}_{\boldsymbol{p}}$ & $\boldsymbol{R}$ & $\boldsymbol{n}=\boldsymbol{f}(\boldsymbol{t})$ & $\boldsymbol{j}$ & Type \\
\hline \hline 7 & 13 & 3 & $1+3 t$ & --- & $p$ \\
\hline 11 & 89 & 9 & $2+3 t$ & 0 & $p$ \\
\hline 13 & 233 & 3 & $2+3 t$ & 1 & $p$ \\
\hline 17 & 1597 & 7 & $3 t$ & 7 & $p$ \\
\hline 19 & 4181 & 1 & $1+3 t$ & 19 & $c$ \\
\hline 23 & 28657 & 7 & $3 t$ & 136 & $p$ \\
\hline 29 & 514229 & 9 & $2+3 t$ & 2448 & $p$ \\
\hline 31 & 1346269 & 9 & $1+3 t$ & 6410 & $C$ \\
\hline 37 & 24157817 & 7 & $1+3 t$ & 15037 & $c$ \\
\hline 41 & 165580141 & 1 & $3 t$ & 788476 & $c$ \\
\hline 43 & 433494437 & 7 & $1+3 t$ & 2064259 & $p$ \\
\hline 47 & 2971215073 & 3 & $1+3 t$ & 14148643 & $p$ \\
\hline 53 & 53316291173 & 3 & $2+3 t$ & 253887100 & $c$ \\
\hline 59 & 956722026041 & 1 & $1+3 t$ & 4555819171 & $c$ \\
\hline
\end{tabular}




\begin{tabular}{|c|r|r|r|r|c|}
\hline $\boldsymbol{p}$ & \multicolumn{1}{|c|}{$\boldsymbol{F}_{\boldsymbol{p}}$} & $\boldsymbol{R}$ & $\boldsymbol{n}=\boldsymbol{f}(\boldsymbol{t})$ & $\boldsymbol{j}$ & Type \\
\hline \hline 61 & 2504730781961 & 1 & $1+3 t$ & 1927289438 & $c$ \\
\hline 67 & 44945570212853 & 3 & $2+3 t$ & 214026524823 & $c$ \\
\hline 71 & 308061521170129 & 9 & $1+3 t$ & 1466959624619 & $c$ \\
\hline 73 & 806515533049393 & 3 & $1+3 t$ & 3840550157378 & $c$ \\
\hline 79 & 14472334024676221 & 1 & $3 t$ & 6891587607982 & $c$ \\
\hline 83 & 99194853094755497 & 7 & $1+3 t$ & 472356443308359 & $p$ \\
\hline 89 & 1779979416004714189 & 9 & $1+3 t$ & 8476092457165305 & $c$ \\
\hline 97 & 83621143489848422977 & 7 & $3 t$ & 3983195921380230 & $c$ \\
\hline 101 & 573147844013817084101 & 1 & $1+3 t$ & 2729275447684843257 & $c$ \\
\hline 103 & 1500520536206896083277 & 7 & $3 t$ & 71453358866699505158 & $c$ \\
\hline 107 & 10284720757613717413913 & 3 & $2+3 t$ & 48974860750541511494 & $c$ \\
\hline
\end{tabular}

Table 7. Fibonacci types $\left(5 \leq F_{p} \leq 10284720757613717413913\right)$

\begin{tabular}{|c|c|c|c|c|}
\hline $\boldsymbol{R}$ & $\mathbf{2 + 3}$ & $\mathbf{3} \boldsymbol{t}$ & $\mathbf{1}+\mathbf{3} \boldsymbol{t}$ & Total \\
\hline \hline 1 & - & - & - & 0 \\
\hline 3 & 1 & - & 2 & 3 \\
\hline 7 & - & 2 & 2 & 4 \\
\hline 9 & 2 & - & - & 2 \\
\hline
\end{tabular}

Table 8. Numbers of $R$

\subsection{Mersenne primes}

The Mersenne numbers, $M_{m}=2^{m}-1$ ( $m$ odd) have been known for centuries with interest centred on which $m$ yield primes [12]. These primes are found in the very large number domain, yet the REDs can only be 1 or 7 ( $2^{p}$ REDs can only be 2 or 8 ). Moreover, in the $n R$ form $n$ always has the form $3 t$.

\section{Final comments}

The largest known primes (Table 9) are expressed in the form

$$
p=2^{m}-1 \text {. }
$$

Since $m$ for these cases falls in class $\overline{1}_{4}\left(m=4 r_{1}+1\right)$, the RED of $2^{m}$ will be 2 [13] so that RED of $2^{m}-1=1$.

\begin{tabular}{|c|c|c|c|}
\hline No. of digits $\times \mathbf{1 0}^{\mathbf{6}}$ & $\boldsymbol{m} \in \overline{\mathbf{1}}_{\mathbf{4}}$ & $\mathbf{2}^{\mathbf{4 r}_{\mathbf{1}} \mathbf{+ 1}}$ & RED of prime \\
\hline \hline 22.3 & 74207281 & 2 & 1 \\
\hline 17.4 & 51885161 & 2 & 1 \\
\hline 13.0 & 43112609 & 2 & 1 \\
\hline 13.0 & 42643801 & 2 & 1 \\
\hline
\end{tabular}

Table 9. REDs of Mersenne primes

These primes can fall in $(n=3 t)$ or $(n=1+3 t)$ sequences, but $n \neq 16+21 j$ (see Table 4$)$. 


\section{References}

[1] Cattani, C., \& Ciancio, A. (2016) On the fractal distribution of primes and prime-indexed primes by the binary image analysis. Physica A: Statistical Mechanics and Its Applications. 460 (1), 222-229.

[2] Lemke Oliver, R. J., \& Soundararajan, K. (2016) Unexpected biases in the distribution of consecutive primes. Preprint. Available online: https://arxiv.org/abs/1603.03720

[3] Leyendekkers, J. V., Shannon, A. G., \& Rybak, J. M. (2007) Pattern Recognition: Modular Rings and Integer Structure. North Sydney: Raffles KvB Monograph No.9.

[4] Leyendekkers, J. V., \& Shannon, A. G. (1998) The characteristics of primes and other integers within Modular Ring $Z_{4}$ and in Class $\overline{1_{4}}$. Notes on Number Theory and Discrete Mathematics, 4 (1), 1-17.

[5] Leyendekkers, J. V., \& Shannon, A. G. (1998) The characteristics of primes and other integers within Modular Ring $Z_{4}$ and in Class 34 . Notes on Number Theory and Discrete Mathematics, 4 (1), 18-37.

[6] Leyendekkers, J. V., \& Shannon, A. G. (2002) Constraints on powers within the modular ring $Z_{4}$ Part 1: Even powers. Notes on Number Theory and Discrete Mathematics, 8 (2), 41-57.

[7] Leyendekkers, J. V., \& Shannon, A. G. (2004) Extensions of Euler's prime generating functions. Notes on Number Theory and Discrete Mathematics, 10 (4), 100-105.

[8] Leyendekkers, J. V., \& Shannon, A. G. (2008) Analysis of primes using REDs (right-enddigits) and integer structure. Notes on Number Theory and Discrete Mathematics, 14 (3), $1-10$.

[9] Leyendekkers, J. V., \& Shannon, A. G. (2008) The identification of rows of primes in the modular ring $Z_{6}$. Notes on Number Theory and Discrete Mathematics, 14 (4), 10-15.

[10] Leyendekkers, J. V., A.G. Shannon, C.K. Wong. 2009) Spectra of primes. Proceedings of the Jangjeon Mathematical Society. 12 (1), 1-10.

[11] Leyendekkers, J. V., \& Shannon, A. G. (2014) Fibonacci Numbers with Prime Subscripts: Digital Sums for Primes versus Composites. Notes on Number Theory and Discrete Mathematics, 20(3), 45-49.

[12] Knott, R. (2011) The Fibonacci Numbers, Available online: http://www. maths . surrey.ac.uk/hosted-sites/R.Knott/Fibonacci/fibtable.html

[13] Leyendekkers, J. V., \& Shannon, A. G. (2005) Fermat and Mersenne Numbers, Notes on Number Theory and Discrete Mathematics, 11 (4), 17-24. 